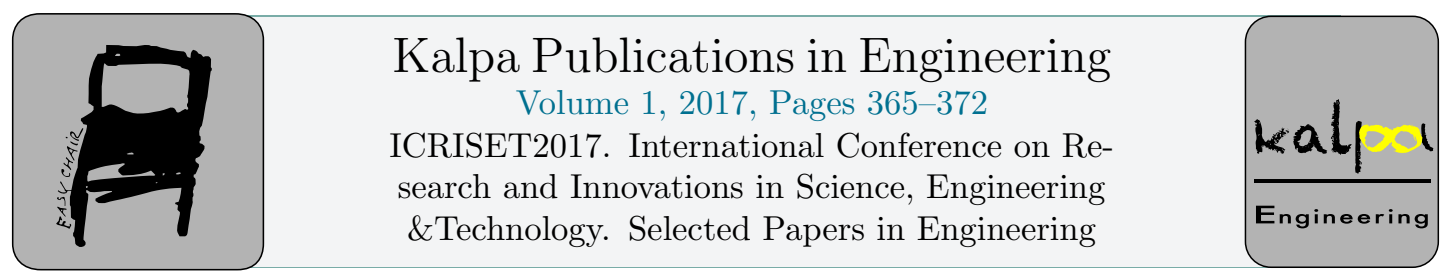

\title{
Linear Sensitivity Analysis and Series Compensation for Transmission Congestion Management
}

\author{
Jalpa H Jobanputra ${ }^{1}$ and Chetan D Kotwal ${ }^{2}$ \\ ${ }^{1}$ Research Scholar, R K University, Rajkot \\ Electrical Engineering Dept., SRICT, Vataria, India \\ ${ }^{2}$ Professor and Head, \\ Electrical Engineering Dept., SVIT, Vasad, India
}

\begin{abstract}
Optimal utilization of transmission system without congestion in the network is most important as congestion can violet the security of the system. Sufficient amount of reactive power support needs to be provided in the system in order to maintain the power flow limits on transmission lines and voltage limits at bus bars. This Paper focuses on analysis of congestion occurrence with N-1 contingencies for line flow limits in case of line and generator outages using linear sensitivity factors and congestion management using series compensation. Generation shift factor and line outage distribution factor are used to find sensitive lines and series compensation in two stages are applied to the most sensitive lines to relieve congestion. Standard IEEE 6 bus test system is used to analyze contingencies and congestion mitigation. All the simulations are performed using power world simulator version 19.0. Mathematical calculations are also performed for the same 6-bus system for validation of results.
\end{abstract}

\section{Introduction}

\subsection{Restructured power system and challenges}

In the recent years, major changes have been introduced into the structure of electric power utilities all over the world. The reason for the introduction of such Major change in existing power utility structure is the scarcity of financial resources available with government and necessity of improving technical and commercial efficiency in the operation of the power system by means of deregulating and restructuring the power industry and opening it up to the private competition. A restructured power system allows the power supply to function competitively, as well as allowing consumers to choose suppliers of electric energy (M. Shahidehpour, 2000)Restructuring in electric power industry has caused an intensive usage of transmission grid.Also, lack of coordination between generation and transmission utilities or as a result of unexpected contingencies such as generation outages, sudden 
increase of load demand, or failure of equipments, causes the transmission system to operate at or beyond one or more transfer limits, the system is said to be congested (P.Bajpai, 2006)

Restructuring in power system has introduced many new technical challenges. Some of the technical challenges are operation of transmission system at optimum level by maintaining all system operational constraints, Ancillary Services Management, Optimal Bidding for a Gencos, Transmission Pricing, enhancement of available transfer capability, determination of total transfer capability, to satisfy most of bilateral transaction keeping in view economy, security and congestion management. Some of the Economical issues associated with deregulation are, active and reactive power spot price and wheeling charges (NERC, 1997) Power system all over the world has been forced to operate in almost their full capacities due to the economical, environmental, and political constraints whereas the almost of electric power that can be transmitted between two buses through a transmission grid is limited by technological and economical constraints. Power flow should not be allowed to increase a level where a random event could cause grid to collapse. Congestion is a situation where the demand for transmission capacity exceeds the transmission network capabilities, which might lead to a violation of network security limits. All the market players try to get the benefit of cheaper source and greater profit margins, which may lead to congestion of certain part of transmission grid. Congestion management is most priority management problem, which the system operator has to face very frequently.

\subsection{Congestion Management Technique}

Congestion Management of transmission network is to reduce the power flow in the congested lines by changing the power flow pattern. Primarily, this process of redispatch can be done by changing generation or modifying load, or both, without compromising the system security and quality of service. In a competitive environment, all the Generation Companies and Distribution Companies plan their transactions ahead of time. However, by the time of implementation of transactions, there may be congestion in some of the transmission lines. Hence, Independent system operator in a competitive electricity market is responsible for serving the necessary actions to ensure that no violations of the grid constraints occur (FERC, 1999) Congestion management approaches are based on issuing orders by the ISO to various parties to reschedule their contracts, re-dispatch generators, use various control devices or compensators, or shed loads in the extreme conditions when these measures are not able to manage the congestion. Basic approach is based on centralized optimization with some form of optimal power flow program or depending upon the control measures executed by the SO for congestion relief (R.D. Christie, 2000)

\section{Linear Sensitivity Analysis}

Several sensitivity factors Viz. Generalized generation distribution factor and generalized line outage distribution factors can be used to find congestion in a particular line. For the same contingencies has to be defined for the components of the system e.g. generators or transmission lines that are not in operation. This situation may arise in the system due to the violation of engineering constraints or maintenance schedule. The effect of this unavoidable situation may be quite severe as it can cause power flow congestion in the line. Transmission lines have thermal limit of carrying power and if the power flow exceeds that limit, which is quite expected during contingency, the line is said to be congested (Sen S., 2011). To avoid such congestion issues preliminary approach is presented of contingency analysis using linear sensitivity factors for realizing congestion in proposed system. Line and bus data of sample 6 bus system is taken from (Bruce, 1984) 


\subsection{Mathematical calculation using sensitivity factors}

The linear sensitivity factors estimate the Changes in different line flows for any particular outage condition without the need of full AC power flow solution. There are two types of sensitivity factors:

1. Generation outage sensitivity factor (GOSF)

2. Line outage sensitivity factor (LOSF)

Steps for GOSF calculations are:

Step-1: Formation of Ybus:

$$
\begin{aligned}
& X 12=0.20 \text { ohm } ; Y 12=\frac{1}{X 12}=\frac{1}{0.2}=-j 5 \mathrm{mho} \\
& X 14=0.20 \mathrm{ohm} ; Y 14=\frac{1}{X 14}=\frac{1}{0.2}=-j 5 \mathrm{mho} \\
& X 15=0.30 \mathrm{ohm} ; Y 15=\frac{1}{X 15}=\frac{1}{0.3}=-j 3.33 \mathrm{mho}
\end{aligned}
$$

As Bus 1 is not connected with Bus $3 \&$ 6.So, $Y 13=0$ mho \& $Y 16=0$ mho

Total Admittance at bus 1 is,

$$
\begin{aligned}
& Y 11=Y 12+Y 13+Y 14+Y 15+Y 16 \\
& Y 11=(-j 5)+0+(-j 5)+(-j 3.33)+0 \\
& Y 11=(-j 13.33) \text { mho }
\end{aligned}
$$

Similarly calculated for each bus and formulated Ybus as below,

$$
\text { Ybus }=\left[\begin{array}{cccccc}
-j 13.33 & j 5.0 & 0 & j 5.0 & j 3.33 & 0 \\
j 5.0 & -j 27.33 & j 4.0 & j 10.0 & j 3.33 & j 5.0 \\
0 & j 4.0 & -j 17.84 & 0 & j 3.84 & j 10 \\
j 5.0 & j 10.0 & 0 & -j 17.5 & j 2.5 & 0 \\
j 3.33 & j 3.33 & j 3.84 & j 2.5 & -j 16.34 & j 3.33 \\
0 & j 5.0 & j 10 & 0 & j 3.33 & -j 18.33
\end{array}\right]
$$

Step-2: Formation of B' Matrix: We will form B' by removing the first row \& column corresponding to swing bus and then taking the negative of the imaginary part of Ybus.

$$
B^{\prime}=\left[\begin{array}{ccccc}
27.33 & -4.0 & -10.0 & -3.33 & -5 \\
-4.0 & 17.84 & 0 & -3.84 & -10.0 \\
-10.0 & 0 & 17.5 & -2.5 & 0 \\
-3.33 & -3.84 & -2.5 & 16.34 & -3.33 \\
-5.0 & -10.0 & 0 & -3.33 & 18.33
\end{array}\right]
$$

Step-3: Formation of X Matrix:X $=\left[\mathrm{B}^{\prime}\right]^{-1,}\left[\begin{array}{ccccc}0.0941 & 0.0805 & 0.0630 & 0.643 & 0.0813 \\ 0.0805 & 0.1659 & 0.0590 & 0.0908 & 0.1290 \\ 0.0630 & 0.0590 & 0.1009 & 0.0542 & 0.0592 \\ 0.0643 & 0.0908 & 0.0542 & 0.1222 & 0.0893 \\ 0.0813 & 0.1290 & 0.0592 & 0.0893 & 0.1633\end{array}\right]$

Step-4: Calculating GOSF, $[\Delta \S]=[\mathrm{XF}][\Delta \mathrm{P}]$, Where $[\Delta \mathrm{P}]=\left[\begin{array}{c}-1 \\ 1 \\ 0 \\ 0 \\ 0 \\ 0\end{array}\right]$

$1^{\text {st }}$ element is consider as (-1) as bus 1 is swing bus and $2^{\text {nd }}$ element is consider as $(-1)$ as calculation of GSF is for outage of generator Remaining element need to be considered as zero. 


$$
[\Delta \S]=\left[\begin{array}{c}
0 \\
0.094125 \\
0.080513 \\
0.062977 \\
0.064343 \\
0.081285
\end{array}\right] \mathrm{a}_{\mathrm{i}}^{1}=\frac{1}{\mathrm{x}_{\mathrm{l}}}\left[\Delta \S_{\mathrm{pm}}^{\mathrm{i}}-\Delta \S_{\mathrm{pn}}^{\mathrm{i}}\right], \Delta \S_{\mathrm{pm}}^{\mathrm{i}}=\text { mth element of }[\Delta \S], \Delta \S_{\mathrm{pn}}^{\mathrm{i}}=\text { nth element of }[\Delta \S]
$$

$\frac{1}{\mathrm{Xl}}=$ Inverse of line reactance, as per the data given

Sample calculation for Bus-2 line 7 as given below:

$$
\mathrm{a}_{\mathrm{i}}^{1}=\frac{1}{\mathrm{x}_{1}}\left[\Delta \oint_{\mathrm{pm}}^{\mathrm{i}}-\Delta \oint_{\mathrm{pn}}^{\mathrm{i}}\right], \mathrm{a}_{2}{ }^{7}=\frac{1}{0.2}[0.080513-0.128954], \mathrm{a}_{2}{ }^{7}=5[0.048439], \mathrm{a}_{2}{ }^{7}=0.31147
$$

Generation shift factor for Bus-2 and 3 are similarly calculated and shown in table no.1 as given below

\begin{tabular}{ccccc}
\hline Line No. & Buses $m \rightarrow n$ & Bus- 1 & Bus-2 & Bus-3 \\
\hline 1 & $1-2$ & 0 & -0.47062 & -0.40256 \\
2 & $1-4$ & 0 & -0.31489 & -0.29487 \\
3 & $1-5$ & 0 & -0.21448 & -0.30256 \\
4 & $2-3$ & 0 & 0.05445 & -0.34156 \\
5 & $2-4$ & 0 & 0.31147 & 0.21539 \\
6 & $2-5$ & 0 & 0.09927 & -0.03418 \\
7 & $2-6$ & 0 & 0.06420 & -0.24221 \\
8 & $3-5$ & 0 & 0.06219 & 0.28898 \\
9 & $3-6$ & 0 & -0.00773 & 0.36949 \\
10 & $4-5$ & $0 ;$ & -0.00341 & -0.07948 \\
11 & $5-6$ & 0 & -0.05647 & -0.12729 \\
\hline
\end{tabular}

Table 1: Generation shift sensitivity factors (GSF)

Step-5: Calculating Line outage distribution factor:

$$
\mathrm{d}_{\mathrm{l}, \mathrm{k}}=\frac{\Delta \mathrm{F}_{\mathrm{i}}}{\mathrm{F}_{\mathrm{k}}^{0}}
$$

$$
\mathrm{d}_{\mathrm{l}, \mathrm{k}}=\text { Line outage distribution factor }
$$

where, Delta $\mathrm{F}_{\mathrm{i}}=$ Change in MW flow of line $\mathrm{l}$ and $\mathrm{F}_{\mathrm{k}}^{0}=$ Intial power flow in line $\mathrm{k}$ (before outage) Let line, 1 be connected between buses I and $\mathrm{j}$.

Delta $\mathrm{f}_{\mathrm{i}}=\frac{1}{\mathrm{x}_{\mathrm{l}}}\left(\Delta \S_{\mathrm{i}}-\Delta \S_{\mathrm{j}}\right)$, Using both above Equation we get

$\mathrm{d}_{\mathrm{l}, \mathrm{k}}=\frac{\text { Delta } \mathrm{F}_{\mathrm{i}}}{\mathrm{F}_{\mathrm{k}}^{0}}, \mathrm{~d}_{\mathrm{l}, \mathrm{k}}=\frac{\frac{1}{\mathrm{x}_{1}}\left(\Delta \S_{\mathrm{i}}-\Delta \S_{\mathrm{j}}\right)}{\mathrm{P}_{\mathrm{mn}}}=\frac{1}{\mathrm{x}_{\mathrm{l}}}\left(\frac{\Delta \S_{\mathrm{i}}}{\mathrm{P}_{\mathrm{mn}}}-\frac{\Delta \S_{\mathrm{i}}}{\mathrm{P}_{\mathrm{mn}}}\right)=\frac{1}{\mathrm{x}_{\mathrm{l}}}\left(\mathrm{B}_{\mathrm{mn}}^{\mathrm{i}}-\mathrm{B}_{\mathrm{mn}}^{\mathrm{j}}\right)$

If $i$ and $j$ are not reference buses, then

$\mathrm{d}_{\mathrm{l}, \mathrm{k}}=\frac{1}{\mathrm{X}_{\mathrm{l}}} \frac{\left(\left(\mathrm{X}_{\mathrm{im}}-\mathrm{X}_{\mathrm{in}}\right) \mathrm{x}_{\mathrm{k}}-\left(\mathrm{X}_{\mathrm{jm}}-\mathrm{X}_{\mathrm{jn}}\right) \mathrm{x}_{\mathrm{k}}\right)}{\mathrm{X}_{\mathrm{k}}-\left(\mathrm{X}_{\mathrm{mn}}+\mathrm{X}_{\mathrm{nn}}-2 \mathrm{X}_{\mathrm{mn}}\right)}$

Where, Line 1 is connected from bus $\mathrm{i}$ to $\mathrm{j}$, Line $\mathrm{k}$ is connected from bus $\mathrm{m}$ to $\mathrm{n}$. A sample calculation is shown below. Consider element d5, 4. This is the factor for line 5, when line 4 faces an outage. Here $1=5$ and $k=4$. Line is connected between 2 and $4(i-j)$ and line 4 between 2 and 3 (m-n).

We use Eq. To get

$$
\begin{aligned}
& \mathrm{d}_{\mathrm{l}, \mathrm{k}}=\frac{\mathrm{X}_{\mathrm{k}}}{\mathrm{X}_{\mathrm{l}}} \frac{\left(\mathrm{X}_{\mathrm{im}}-\mathrm{X}_{\mathrm{in}}-\mathrm{X}_{\mathrm{jm}}+\mathrm{X}_{\mathrm{jn}}\right)}{\mathrm{X}_{\mathrm{k}}-\left(\mathrm{X}_{\mathrm{mm}}+\mathrm{X}_{\mathrm{nn}}-2 \mathrm{X}_{\mathrm{mn}}\right)} \mathrm{d}_{5,4}=\frac{\mathrm{X}_{4}}{\mathrm{X}_{5}} \mathrm{X} \frac{\left(\mathrm{X}_{22}-\mathrm{X}_{23}-\mathrm{X}_{42}+\mathrm{X}_{43}\right)}{\mathrm{X}_{4}-\left(\mathrm{X}_{22}+\mathrm{X}_{33}-2 \mathrm{X}_{23}\right)} \\
& \mathrm{X}_{\mathrm{F}}=\left[\begin{array}{llllll}
0 & 0 & 0 & 0 & 0 & 0 \\
0 & 0.0941 & 0.0805 & 0.0630 & 0.643 & 0.0813 \\
0 & 0.0805 & 0.1659 & 0.0590 & 0.0908 & 0.1290 \\
0 & 0.0630 & 0.0590 & 0.1009 & 0.0542 & 0.0592 \\
0 & 0.0643 & 0.0908 & 0.0542 & 0.1222 & 0.0893 \\
0 & 0.0813 & 0.1290 & 0.0592 & 0.0893 & 0.1633
\end{array}\right] \mathrm{d}_{5,4}=\frac{0.25}{0.1} \mathrm{X} \frac{(0.0941-0.0630-0.0805+0.0590)}{0.25-(0.0941+0.1659-2 \mathrm{x} 0.0805)}=0.159
\end{aligned}
$$

Line-outage distribution factors of all the other lines are similarly calculated and shown in table 2 


\begin{tabular}{cccccccccccc}
\hline $\mathrm{l} / \mathrm{k}$ & $\mathrm{k}=1$ & $\mathrm{k}=2$ & $\mathrm{k}=3$ & 4 & 5 & 6 & 7 & 8 & 9 & 10 & 11 \\
\hline $\mathrm{l}=1$ & 0 & 635 & 543 & -112 & -503 & -210 & -122 & -137 & 14 & 10 & 132 \\
$\mathrm{l}=2$ & 595 & 0 & 457 & -33 & 612 & -61 & -35 & -40 & 4 & -327 & 39 \\
$\mathrm{l}=3$ & 405 & 365 & 0 & 146 & -109 & 272 & -158 & 177 & -17 & 317 & -170 \\
$\mathrm{l}=4$ & -103 & -32 & 178 & 0 & 124 & 226 & -466 & -400 & -525 & 171 & 132 \\
$\mathrm{l}=5$ & -588 & 765 & -170 & 159 & 0 & 297 & 172 & 193 & -19 & -673 & -186 \\
$\mathrm{l}=6$ & -188 & -59 & 325 & 221 & 226 & 0 & 239 & 269 & -26 & 311 & -258 \\
$\mathrm{l}=7$ & -121 & -38 & 210 & 507 & 146 & 267 & 0 & -199 & 584 & 201 & 443 \\
$\mathrm{l}=8$ & -118 & -37 & 204 & -375 & 142 & 258 & -172 & 0 & 475 & 195 & -425 \\
$\mathrm{l}=9$ & 15 & 5 & -25 & -624 & -17 & -32 & 638 & 601 & 0 & -24 & 557 \\
$\mathrm{l}=10$ & 7 & -235 & 287 & 126 & -387 & 235 & 137 & 153 & -15 & 0 & -147 \\
$\mathrm{l}=11$ & 107 & 34 & -184 & 117 & -128 & -265 & 362 & -401 & 416 & -177 & 0 \\
\hline
\end{tabular}

Table 2 Line outage distribution factor (LODF)

\section{Simulation and results}

Similar sample 6 bus system is used to perform contingency analysis using sensitivity factors. Fig no. 1 shows GSF/TLR sensitivity analysis with the outage of a generator no. 3 using PWS (PWS, 2013)and table 3 shows the generation shift factors for the same.

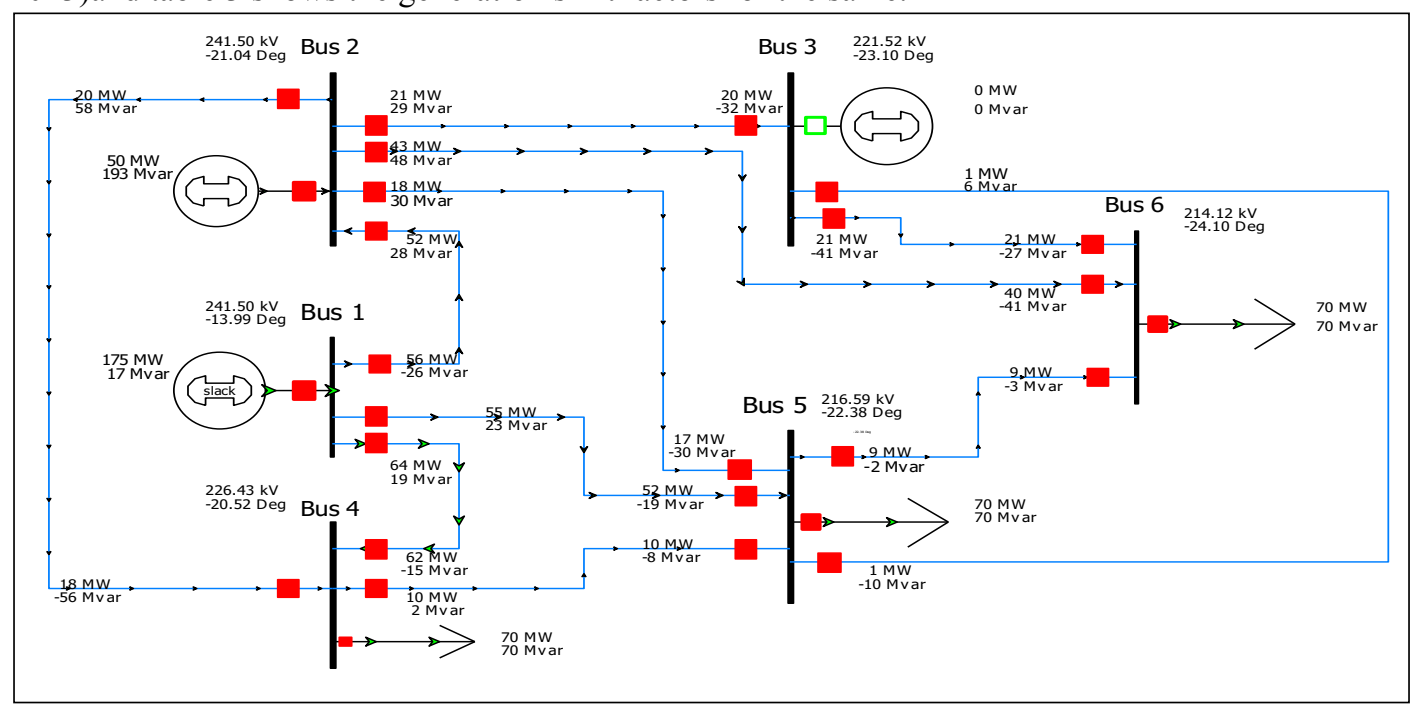

Figure 1: Simulation circuit of sample 6 bus system for GSF/TLR analysis for outage of generator 3

\begin{tabular}{cccccccccccc}
\hline Bus No & $1>>2$ & $1>>4$ & $1>>5$ & $2>>3$ & $2>>4$ & $2>>5$ & $2>>6$ & $3>>5$ & $3>>6$ & $4>>5$ & $5>>6$ \\
\hline Bus 1 & 0 & 0 & 0 & 0 & 0 & 0 & 0 & 0 & 0 & 0 & 0 \\
Bus 2 & -470 & -314 & -214 & 54 & 311 & 99 & 64 & 62 & -7 & -3 & -56 \\
Bus 3 & -402 & -294 & -302 & -341 & 215 & -34 & -242 & 289 & 369 & -79 & -127 \\
Bus 4 & -314 & -504 & -180 & 16 & -379 & 29 & 18.9 & 18 & -2 & 116 & -16 \\
Bus 5 & -321 & -271 & -407 & -105 & 101 & -192 & -124 & -120 & 15 & -169 & 109.6 \\
Bus 6 & -406 & -296 & -297 & -190 & 220 & -26 & -410 & 152 & -343 & -75.2 & -246.7 \\
\hline
\end{tabular}

$\mathbf{x ~ 1 0}^{-3}$ Table 3 Simulation results for GSF for outage of gen-3 
Line outage distribution factor sensitivity analysis is similarly performed on same system to analyze all possible contingencies and finally most sensitive lines considering total MVA flows. Fig 2 shows LODF analysis for outage of line 1and table 4 represents simulation results of LODF for outage of all 11 lines one by one.

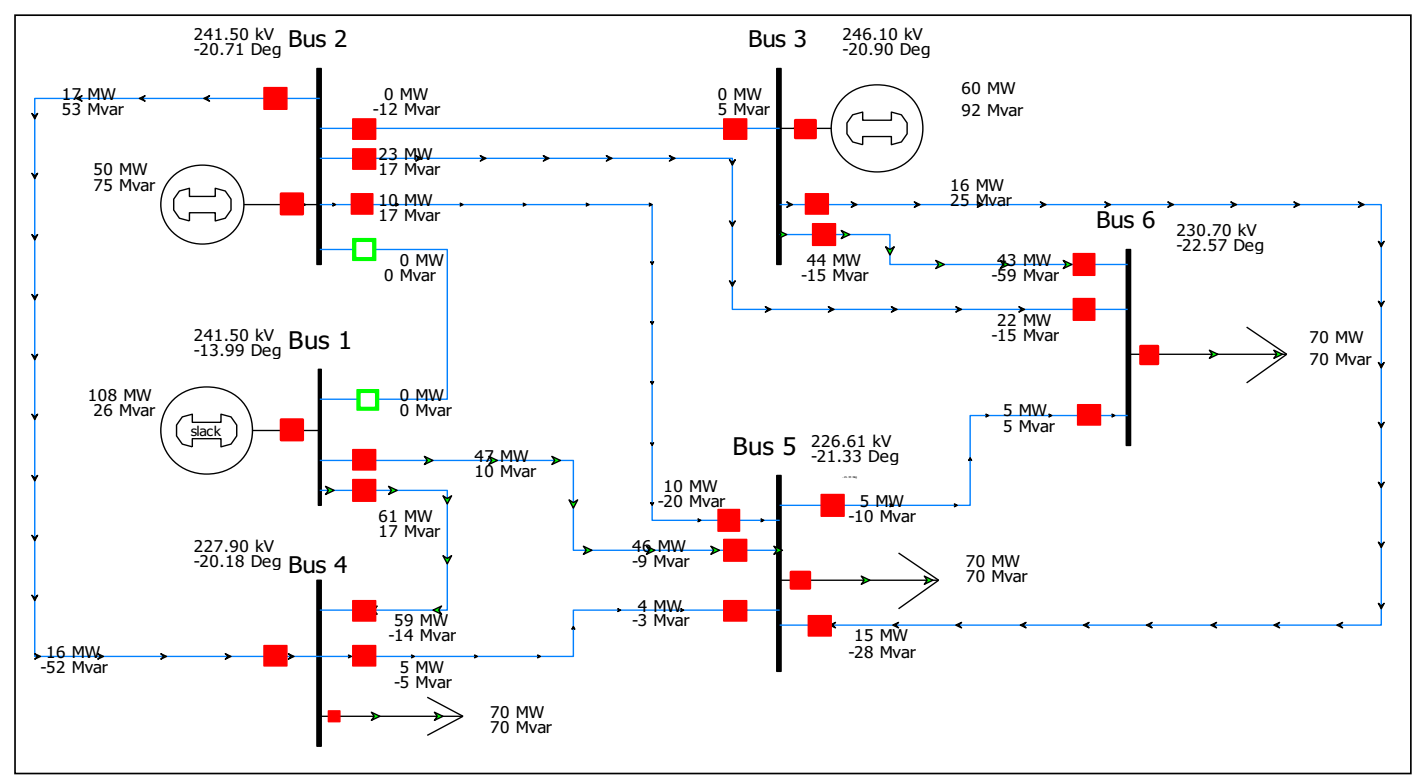

Figure 2: Simulation circuit of 6-bus sample system for LODF (outage of 1-2)

\begin{tabular}{|c|c|c|c|c|c|c|c|c|c|c|c|c|}
\hline \multicolumn{3}{|c|}{$\begin{array}{l}\text { LineFro To } \\
\text { No. } . \mathrm{m}\end{array}$} & $\begin{array}{c}1-2 \\
\text { OUT }\end{array}$ & $\begin{array}{c}1-4 \\
\text { OUT }\end{array}$ & $\begin{array}{c}1-5 \\
\text { OUT }\end{array}$ & $\begin{array}{c}2-3 \\
\text { OUT }\end{array}$ & $\begin{array}{c}2-4 \\
\text { OUT }\end{array}$ & $\begin{array}{c}2-6 \\
\text { OUT }\end{array}$ & $\begin{array}{c}3-5 \\
\text { OUT }\end{array}$ & $\begin{array}{c}3-6 \\
\text { OUT }\end{array}$ & $\begin{array}{c}4-5 \\
\text { OUT }\end{array}$ & $\begin{array}{c}5-6 \\
\text { OUT }\end{array}$ \\
\hline \multicolumn{13}{|c|}{ Bus } \\
\hline 1 & 1 & 2 & 0 & -63.5 & -54.3 & 11.3 & 50.3 & 12.2 & 13.7 & -1.3 & -1 & -13.2 \\
\hline 2 & 1 & 4 & -59.5 & 0 & -45.7 & 3.3 & -61.2 & 3.6 & 4 & -0.4 & 32.7 & -3.9 \\
\hline 3 & 1 & 5 & -40.5 & -36.5 & 0 & -14.6 & 10.9 & -15.8 & -17.7 & 1.7 & -31.7 & 17 \\
\hline 4 & 2 & 3 & 10.3 & 3.2 & -17.8 & 0 & -12.4 & -46.6 & 40 & 52.5 & -17.1 & -13.2 \\
\hline 5 & 2 & 4 & 58.8 & -76.5 & 17.1 & -15.9 & 0 & -17.2 & -19.3 & 1.9 & 67.3 & 18.6 \\
\hline 6 & 2 & 5 & 18.8 & 5.9 & -32.5 & -22.1 & -22.6 & -23.9 & -26.8 & 2.6 & -31.1 & 25.8 \\
\hline 7 & 2 & 6 & 12.1 & 3.8 & -21 & -50.7 & -14.6 & 0 & 19.9 & -58.4 & -20.1 & -44.3 \\
\hline 8 & 3 & 5 & 11.7 & 3.7 & -20.4 & 37.5 & -14.2 & 17.2 & 0 & -47.5 & -19.5 & 42.5 \\
\hline 9 & 3 & 6 & -1.5 & -0.5 & 2.5 & 62.5 & 1.8 & -63.8 & -60 & 0 & 2.4 & -55.7 \\
\hline 10 & 4 & 5 & -0.6 & 23.5 & -28.6 & -12.6 & 38.8 & -13.6 & -15.3 & 1.5 & 0 & 14.7 \\
\hline 11 & 5 & 6 & -10.7 & -3.4 & 18.5 & -11.7 & 12.9 & -36.2 & 40.1 & -41.6 & 17.7 & 0 \\
\hline
\end{tabular}

Table 4 Simulation results for LODF sensitivity analysis

Series compensation is provided on the most sensitive lines based on contingency analysis. Fig 3 shows simulation circuit with compensation stage 1 in which even in case of outage of line number 1 (1-2), all other line flows are within their limits. Similarly, all possible contingencies are analyzed and represented in table no.5 with compensation. 


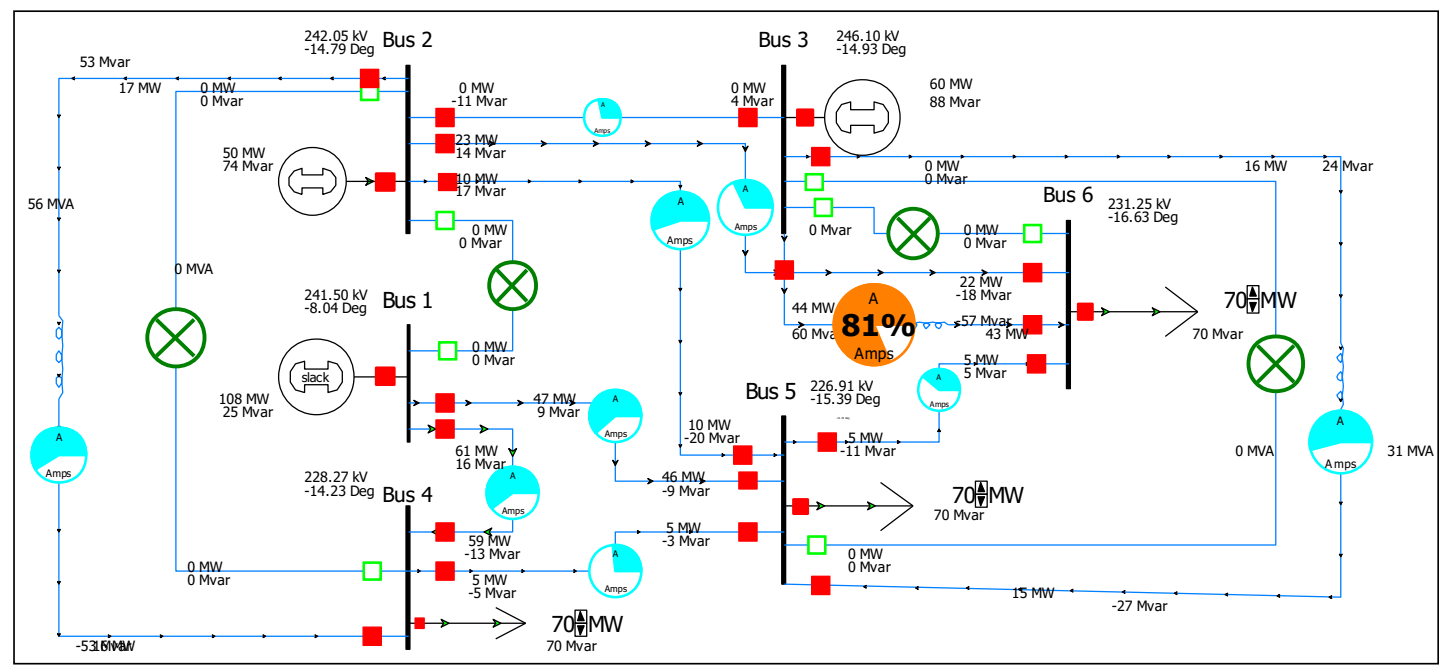

Figure 3: Simulation circuit with compensation (outage of 1-2)

\begin{tabular}{|c|c|c|c|c|c|c|c|c|c|c|c|c|}
\hline \multicolumn{2}{|c|}{ Contingency } & $1>>2$ & $1>>4$ & $1>>5$ & $2>>3$ & $2>>4$ & $2>>5$ & $2>>6$ & $3>>5$ & $3>>6$ & $4>>5$ & $5>>6$ \\
\hline \multicolumn{13}{|c|}{ Normal } \\
\hline \multicolumn{2}{|c|}{ Power flow } & 32.58 & 48 & 37.33 & 12.59 & 56.71 & 21.83 & 29.02 & 31.71 & 74.85 & 6.41 & 9.8 \\
\hline 1 & $1>>2$ & - & 62.74 & 48.31 & 10.68 & 59.09 & 22.54 & 28.78 & 30.99 & 74.1 & 6.54 & 11.55 \\
\hline 2 & $1>>4$ & 65.1 & - & 53.61 & 23.52 & 92.04 & 15.57 & 23.78 & 50.47 & 86.54 & 14.34 & 14.25 \\
\hline 3 & $1>>5$ & 53.33 & 65.92 & - & 21.84 & 53.98 & 33.03 & 35.19 & 43.22 & 83.84 & 14.42 & 15.20 \\
\hline 4 & $2>>3$ & 30.69 & 48.95 & 38.04 & - & 54.39 & 22.58 & 30.25 & 32.48 & 76.62 & 6.96 & 10.00 \\
\hline 5 & $2>>4$ & 28.98 & 90.87 & 36.16 & 9.95 & - & 36.15 & 41.05 & 34.06 & 67.29 & 19.72 & 12.81 \\
\hline 6 & $2>>5$ & 32.18 & 47.32 & 43.47 & 11.54 & 63.66 & - & 35.82 & 38.14 & 75.93 & 10.01 & 14.54 \\
\hline 7 & $2>>6$ & 35.41 & 37.88 & 38.53 & 29.13 & 87.06 & 17.25 & - & 50.33 & 90.86 & 13.4 & 19.09 \\
\hline 8 & $3>>5$ & 28.72 & 50.32 & 45.02 & 15.23 & 58.96 & 31.27 & 27.97 & - & 87.88 & 7.46 & 16.65 \\
\hline 9 & $3>>6$ & 34.73 & 60.14 & 46.89 & 32.24 & 45.78 & 23.7 & 81.07 & 54.67 & - & 7.56 & 28.03 \\
\hline 10 & $4>>5$ & 32.47 & 47.69 & 39.08 & 13.97 & 57.53 & 24.31 & 30.54 & 33.12 & 76.2 & - & 10.37 \\
\hline 11 & $5>>6$ & 32.79 & 48.67 & 37.89 & 13.41 & 57.16 & 24.91 & 29.87 & 34.05 & 73.65 & 5.87 & - \\
\hline 12 & G1 & 55.2 & 69.32 & 49.11 & 33.01 & 35.74 & 11.73 & 23.86 & 43.7 & 90.8 & 8.66 & 12.02 \\
\hline 13 & G2 & 68.35 & 90.91 & 79.63 & 33.33 & 28.78 & 27.01 & 58.84 & 2.84 & 34.29 & 13.26 & 11.93 \\
\hline
\end{tabular}

Table 5 Simulation results with compensation stage 1

For stage 2 compensation variable capacitor is connected with the same lines as shown in fig 3 and change in total MVA flow obtained for each outage are tabulated in table no.6

\begin{tabular}{cccccc}
\hline Sr No. & Outages & $\begin{array}{c}\text { Change in } \\
\text { total MVA flow }\end{array}$ & $\begin{array}{c}\text { Sr } \\
\text { No. }\end{array}$ & Outages & $\begin{array}{c}\text { Change in } \\
\text { total MVA flow }\end{array}$ \\
\hline 1 & $\begin{array}{c}\text { Normal system } \\
\text { flow }\end{array}$ & 361 & 8 & Line out 7 & 419 \\
2 & Line out 1 & 355 & 9 & Line out 8 & 369 \\
3 & Line out 2 & 439 & 10 & Line out 9 & 415 \\
4 & Line out 3 & 420 & 11 & Line out 10 & 365 \\
5 & Line out 4 & 351 & 12 & Line out 11 & 358 \\
6 & Line out 5 & 377 & 13 & G2 Out & 433 \\
7 & Line out 6 & 373 & 14 & G3 Out & 449 \\
\hline
\end{tabular}

Table 6 Simulation results with compensation stage 2 


\section{Conclusion}

In this paper using linear sensitivity factors all the possible contingencies were analyzed and most sensitive lines were found out those contributes in causing congestion in system. Static capacitors were connected in series on sensitive lines which results in reduction of MVA flows of lines during all contingencies and hence mitigation in congestion. Compared to static capacitors, Variable capacitors can provide more tuned compensation to the system.

\section{Acknowledgment}

We gratefully acknowledge the support provided by Shroff S R Rotary Institute of Chemical Technology for providing platform for our research. We are also thankful to RK University and Associate director of FDSR for their support.

\section{References}

Shahidehpour, H. Hamin, and Li (2000) , "Market operations in electric power systems- Forecasting, Scheduling, and Risk Management", The Institute of Electrical and Electronics Engineers, Inc., New York, A John Wiley \& Sons, Inc., Publication.

Bajpai,P and Singh,S N (2006) "An Electric Power Trading Model for Indian Electricity Market”,Power Engineering Society General Meeting, IEEE.

NERC (1997, March) Final Report: Interconnected Operation Services Working Group (IOSWG), Defining Interconnected Operation Services under Open Access

Federal Energy Regulatory Commission (FERC) (1999, December), Regional Transmission Organizations, Washington, DC

Christie, R D ,Wollenberg,, B F and Wangstien, I (2000,February) In Proceedings of Transmission management in the deregulated environment, $(\mathrm{pp}$ 170-195), IEEE 88

Sen, S, Chanda, S, Sengupta, S and Chakrabarti A,(2011) "Differential Evolution based MultiObjective Optimization of a Deregulated Power Network under Contingent State, 3(1) (pp 118)

Wood Allen,J andWollenberg, F Bruce,(1984) "Power Generation, Operation and Control", ISBN 10: 0471091820 / ISBN 13: 9780471091820 , John Wiley \& Sons

Power World Simulator, Power World Corporation,(2013) version 19.0, Evaluation and University Educational Use, URBANA, IL 61801 